\title{
Article \\ The appetite of patients 12 months after intensive care unit dis- charge is associated with depression
}

\author{
Takeshi Unoki ${ }^{*}$, Aki Sasaki1,12, Hideaki Sakuramoto², Sakura Uemura ${ }^{3}$, Takahiro Tsujimoto4, Takako Yamaguchi ${ }^{5}$, \\ Yuko Shiba', Mayumi Hino", Tomoki Kuribara1, Yuko Fukuda ${ }^{8}$, Takumi Nagao', Mio Kitayama ${ }^{10}$, Masako Shi- \\ rasaka11, Junpei Haruna $^{12}$, Yosuke Satoi ${ }^{13}$, Yoshiki Masuda ${ }^{14}$, on behalf of the SMAP-HoPe Study Project
}

${ }^{1}$ Department of Acute and Critical Care Nursing, School of Nursing, Sapporo City University,

Chuo-ku, Sapporo, Hokkaido, Japan

${ }^{2}$ Department of Adult Health Nursing, College of Nursing, Ibaraki Christian University, Hitachi, Ibaraki, Japan

${ }^{3}$ Emergency and Critical Care Medical Center, Osaka City General Hospital, Osaka, Japan

${ }^{4}$ Nursing Practice and Career Support Center, Nara Medical University Hospital, Kashihara City, Nara, Japan

${ }^{5}$ Intensive Care Unit, Nippon Medical School Musashikosugi Hospital, Kawasaki, Kanagawa, Japan

${ }^{6}$ Intensive Care Unit, University of Tsukuba Hospital, Tsukuba, Ibaraki, Japan

${ }^{7}$ Intensive Care Unit, Tohoku Medical and Pharmaceutical University Hospital, Sendai, Miyagi, Japan

${ }^{8}$ Intensive Care Unit, Jichi Medical University Hospital, Yakushiji Shimotsuke-shi, Tochigi, Japan

${ }^{9}$ Intensive Care Unit, Sakakibara Heart Institute, Fuchu-shi, Tokyo, Japan

${ }^{10}$ Nursing Department Heart Center, Kanazawa Medical University Hospital, Uchinada, Ishikawa, Japan

${ }^{11}$ Intensive Care Unit \& Cardiac Care Unit, Japanese Red Cross Fukuoka Hospital, Fukuoka, Japan

${ }^{12}$ Intensive Care Unit, Sapporo Medical University Hospital, Sapporo, Hokkaido, Japan

${ }^{13}$ Intensive Care Unit, Naha City Hospital, Naha, Okinawa, Japan

${ }^{14}$ Department of Intensive Care Medicine, School of Medicine, Sapporo Medical University, Sapporo, Hokkaido, Japan

* Correspondence: t.unoki@suc.ac.jp; Tel.: (optional; include country code; if there are multiple corresponding authors, add author initials)

Abstract: Appetite loss is common in older patients and an independent risk factor for sarcopenia, which is associated with high mortality. However, few studies have explored the phenomenon of appetite loss after discharge from the intensive care unit (ICU). Therefore, we aimed to describe the prevalence of appetite loss and the relationship between appetite loss and depression in patients living at home 12 months after intensive care. We conducted a post hoc analysis, which was a published ambidirectional study examining post-intensive care syndrome at 12 months after discharge. We included patients aged $>65$ years. The Short Nutritional Assessment Questionnaire (SNAQ) and Hospital Anxiety Depression Scale (HADS) were used for the analysis. Descriptive statistics and a multilevel generalized linear model were used to clarify the relationship between appetite loss and depression. Data from 468 patients were analyzed. The defined appetite loss was $25.4 \%$ (95\%CI, 21.5-29.4). High severity of depression was an independent factor for high probability of appetite loss (OR, 1.2; 95\%CI, 1.14-1.28; $\mathrm{p}=0.00)$. Poor appetite is common 12 months after intensive care and is associated with the severity of depression.

Keywords: anorexia; aged; intensive care; depression

\section{Introduction}

Decreased appetite in the process of aging is common [1]. The prevalence of appetite loss is $10.7 \%$ among community-dwelling elders [2]. Additionally, the prevalence of 
appetite loss has increased to $21.2 \%$ in the older population, including those admitted to hospitals or nursing homes [3].

An observational cohort study suggested that appetite loss is an independent risk factor for sarcopenia (OR, 1.42; 95\%CI, 1.06-1.92)[2]. A recent systematic review indicated that sarcopenia in the older population is associated with high mortality [4]. Thus, appetite loss in this population is an important issue.

However, little is known about the studies exploring appetite loss after discharge from the intensive care unit (ICU). An observational cohort study reported a high prevalence of appetite loss in patients discharged from the ICU compared to in age-and sexmatched non-ICU-treated patients [5]. However, this study has some limitations. First, the study did not use validated appetite screening tools. Second, the period from ICU discharge to the point of measurement of appetite varied. Therefore, it is worthwhile to investigate the prevalence of poor appetite in post-intensive care patients using a validated screening tool.

A previous study indicated that depression is associated with poor appetite in community-dwelling older individuals [6]. Depression is a serious issue in patients after intensive care. A systematic review suggested that $29 \%$ of the patients experienced depression 12 months after ICU discharge [7]. To the best of our knowledge, no study has examined the relationship between depression and poor appetite in this population after intensive care.

Therefore, this study aimed to describe appetite loss using validated screening tools, to examine whether depression is related to appetite loss, and to identify the risk factors for appetite loss in older patients 12 months after ICU discharge.

\section{Materials and Methods}

\subsection{Study Design}

This study was a post-hoc analysis of a published ambidirectional study examining post-intensive care syndrome 12 months after discharge (SMAP-HoPe study) [8] conducted in 12 ICUs in Japan. This study focused on appetite in the older cohort.

Briefly, we conducted an ambidirectional study. Eligible patients were those who stayed in the ICU for at least 3 nights between October 2019 and July 2020 and were living at home for 12 months after discharge. The recruitment process continued every month until the specified sample size was reached. The exclusion criteria included central nervous disease, severe dementia, not living at home, and death 12 months after intensive care. After screening the medical chart, we made telephone calls to the candidate patients to clarify if they met the exclusion criteria. After confirming that the patients met the criteria, we sent a survey set including questionnaires regarding mental health and a Japanese-translated simplified nutritional appetite questionnaire (J-SNAQ) at 12 months after intensive care. The detailed recruitment process, study design, and characteristics of each institution has already been published [8]. A total of 778 participants living at home 12 months after intensive care were included in the previous study[8].

\subsection{Participants}

In this study, we included participants over 65 years of age among the 778 participants in the SMAP-HoPe study.

\subsection{Variables and Instruments}

Variables including the Acute Physiology and Chronic Score II (APACHE II), diagnosis at ICU admission, ICU length of stay, and hospital length of stay were retrospectively recorded by a nurse researcher in each ICU. The Simplified Nutritional Appetite Questionnaire (SNAQ) and the Hospital Anxiety and Depression Scale (HADS) surveys were administered 12 months after intensive care. 
The SNAQ has been widely used to assess appetite and has been well validated [9]. An SNAQ score of $<14$ identified significant weight loss. In subjects aged $>60$ years, the sensitivity and specificity of the SNAQ for $10 \%$ weight loss were $83.3 \%$ and $77.6 \%$, respectively [9]. The Japanese-translated SNAQ is reported to have sufficient validity and reliability for the $\geq 65$ years community-dwelling population [10]. We defined poor appetite as $<14$ on the SNAQ according to previous studies $[9,11]$.

The HADS is a commonly used, valid, and reliable questionnaire for assessing the degree of anxiety and depressive symptoms in outpatients [12] and critically ill patients [13]. It has also been translated into Japanese, and its Japanese version has good reliability and validity [14]. The HADS consists of an anxiety subscale and a depression subscale, and each subscale has seven items rated on a scale of 0 to 3 , with a total score of 0 to 21 . Half of the items relate to anxiety symptoms, and the rest, to depressive symptoms. High correlations between HADS scores and psychiatric interview diagnoses of anxiety and depression have been reported (Spearman's correlation: $\mathrm{r}=0.70$ for anxiety severity, $\mathrm{r}=$ 0.74 for depression severity) [14]. In the Japanese version of the HADS, a score of 8 or higher was defined as substantial anxiety or depression [14], and we followed this definition. In this study, the depression subscale of the HADS is referred to as HADS-D. Digestive disease was defined as any digestive disease requiring intensive care.

\subsection{Statistical Analysis}

Because this was a secondary analysis, the sample size was not calculated. Continuous variables and ordinary variables were presented as median and interquartile range (IQR). Nominal variables were presented as percentages. The Kruskal-Wallis test or Fisher's exact test was used to compare the two variables.

To clarify the relationship between poor appetite and severity of depression in the population, a multilevel generalized linear model with binomial family and logit links was used. The outcome variable was poor appetite ( $<14$ on the SNAQ). The covariates included digestive disease, depression, and malignancy at ICU admission.

The missing items of the SNAQ were not imputed because they were considered missing completely at random. Thus, we excluded participants with missing items on the SNAQ. The missing values of the HADS were imputed using the "half rule," which means that if half of the subscales were responded to, the mean value was imputed [15]. R software 4.0.2 (R Foundation for Statistical Computing, 2020) and Stata/IC16 (Stata Corp, TX) were used for analysis.

\section{Results}

\subsection{Characteristics of Participants}

Of the 501 older participants, 33 were excluded because of missing items on the SNAQ; 47 patients had partially missing HADS scores, all of which were substituted using the above method [15]. Thus, 468 participants were included in the analysis. The flow diagram is shown in Figure 1. 


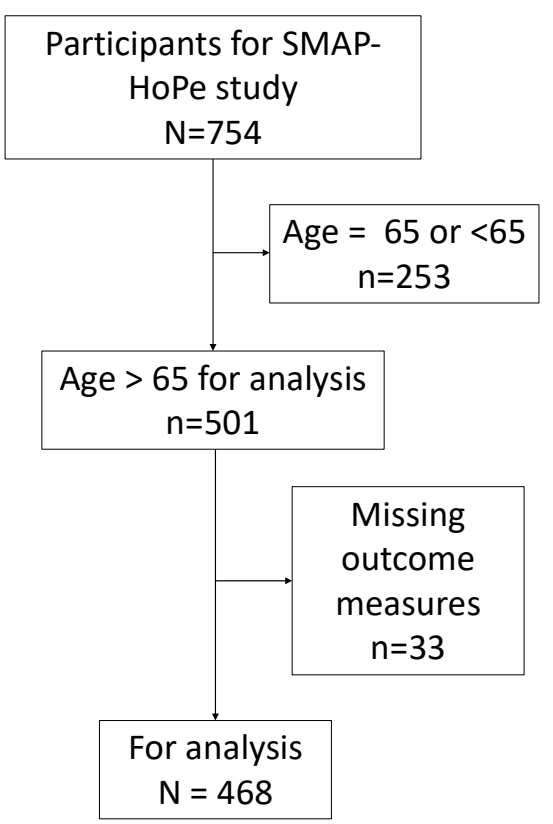

Figure 1. Flow diagram of this study.

The main characteristics of the study population are shown in Table 1 . The median age of the included patientss was 75 years, and approximately $70 \%$ of them were men. Half of the patients were scheduled to undergo surgery. The most frequent reason for ICU admission was cardiovascular surgery. The J-SNAQ score was $14.2 \pm 1.6$, and the prevalence of poor appetite was $25.4 \%$ (95\%CI, 21.5-29.4). A histogram of the SNAQ data is shown in Figure 2.

Table 1. The main characteristics data of the study

\begin{tabular}{|c|c|c|}
\hline Variables & & $\mathrm{N}=468$ \\
\hline Age (years), median [IQR] & & $75.0[71.0,80.0]$ \\
\hline Female, n (\%) & & $142(30.3)$ \\
\hline Elective surgery, n (\%) & & $236(50.4)$ \\
\hline \multicolumn{3}{|l|}{ Diagnosis at admission, $\mathrm{n}(\%)$} \\
\hline & CV surgery & $218(46.6)$ \\
\hline & CHF/AMI/Arry & $72(15.4)$ \\
\hline & Sepsis & $49(10.5)$ \\
\hline & $\begin{array}{l}\text { Abdominal Sur- } \\
\text { gery }\end{array}$ & $41(8.8)$ \\
\hline
\end{tabular}




\begin{tabular}{ccc} 
& $\begin{array}{c}\text { Others } \\
\text { Acute Respira- } \\
\text { tory Failure } \\
\text { Aortic Dissection } \\
\text { (Conservative } \\
\text { Treatment) }\end{array}$ & $19(4.1)$ \\
& ENT & $16(3.4)$ \\
Other Surgery & $15(3.2)$ \\
Trauma & $8(1.7)$ \\
APACHE II, median [IQR] & $8(1.7)$ \\
MV use, $\mathrm{n}(\%)$ & $410(87.6)$ \\
MV (days), median [IQR] & & $15.00[11.0,20.0]$ \\
Delirium, $\mathrm{n}(\%)$ & $324(69.2)$ \\
Malignancy, $\mathrm{n}(\%)$ & $2.00[0.0,3.0]$ \\
ICU LOS, median [IQR] & $122(26.1)$ \\
Hospital LOS, median [IQR] & $94(20.1)$ \\
\hline
\end{tabular}

$\mathrm{IQR}$, interquartile range; $\mathrm{CV}$, cardiovascular; $\mathrm{CHF} / \mathrm{AMI} / \mathrm{Arrhy}$, congestive heart failure/acute myocardial infarction/arrhythmia; ENT, ear nose throat; APACHE II, Acute Physiology and Chronic Health Evaluation II; MV, mechanical ventilation; ICU, intensive care unit; LOS, length of stay

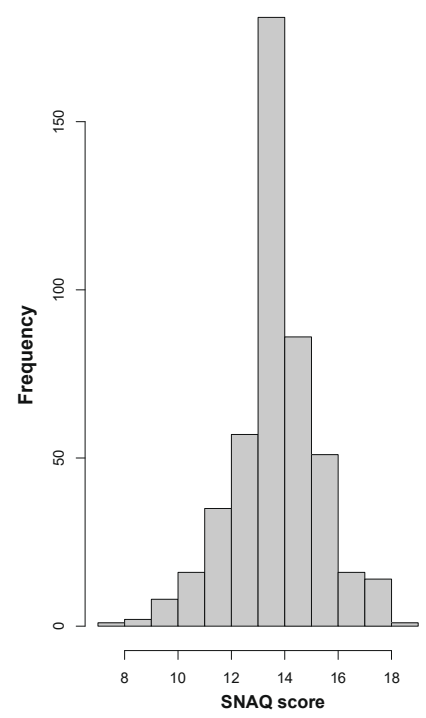

Figure 2. Histogram of scores on the Simplified Nutritional Assessment Questionnaire

\subsection{Characteristics of Participants with or without Poor Appetite}


Table 2 shows a comparison of characteristics between groups, i.e., patients without poor appetite and those with poor appetite. The proportion of patients with malignancy at ICU admission at a risk of weight loss was significantly higher than of those without malignancy. Age, sex, severity of illness, proportion of subjects with digestive illness, and proportion of patients on mechanical ventilation were comparable between the two groups.

Table 2. Comparison of characteristics between groups

\begin{tabular}{|c|c|c|c|}
\hline Variables & $\begin{array}{c}\text { Without poor appe- } \\
\text { tite, } n=349\end{array}$ & $\begin{array}{c}\text { With poor appetite, } \\
n=119\end{array}$ & $\mathrm{P}$ value \\
\hline Age (years), median [IQR] & $75.0[71.0,80.0]$ & $75.0[70.0,79.5]$ & 0.855 \\
\hline Male (\%) & $244(69.9)$ & $82(68.9)$ & 0.908 \\
\hline Elective Surgery, n (\%) & $174(49.9)$ & $62(52.1)$ & 0.75 \\
\hline Diagnosis at admission, $\mathrm{n}(\%)$ & & & 0.83 \\
\hline CV surgery & $165(47.3)$ & $53(44.5)$ & \\
\hline CHF/AMI/Arry & $58(16.6)$ & $14(11.8)$ & \\
\hline Sepsis & $35(10.0)$ & $14(11.8)$ & \\
\hline $\begin{array}{l}\text { Abdominal Sur- } \\
\text { gery }\end{array}$ & $27(7.7)$ & $14(11.8)$ & \\
\hline Others & $17(4.9)$ & $5(4.2)$ & \\
\hline $\begin{array}{l}\text { Acute Respira- } \\
\text { tory Failure }\end{array}$ & $14(4.0)$ & $5(4.2)$ & \\
\hline ENT & $12(3.4)$ & $4(3.4)$ & \\
\hline $\begin{array}{l}\text { Aortic Dissec- } \\
\text { tion (Conserva- } \\
\text { tive Treatment) }\end{array}$ & $10(2.9)$ & $5(4.2)$ & \\
\hline Other Surgery & $6(1.7)$ & $2(1.7)$ & \\
\hline Trauma & $5(1.4)$ & $3(2.5)$ & \\
\hline Digestive disease, n (\%) & $39(11.2)$ & $19(16.0)$ & 0.197 \\
\hline APACHE II, median [IQR] & $15.0[11.0,20.0]$ & $15.00[11.0,20.0]$ & 0.815 \\
\hline MV use, n (\%) & $240(68.8)$ & $84(70.6)$ & 0.732 \\
\hline MV (days), median [IQR] & $2.0[0.0,3.0]$ & $2.00[0.0,3.0]$ & 0.611 \\
\hline Delirium, $\mathrm{n}(\%)$ & $96(27.5)$ & $26(21.8)$ & 0.276 \\
\hline Malignancy, n (\%) & $62(17.8)$ & $32(26.9)$ & 0.035 \\
\hline ICU LOS, median [IQR] & $5.0[4.0,7.0]$ & $5.00[4.0,7.0]$ & 0.981 \\
\hline Hospital LOS, median [IQR] & $29.0[20.0,42.00]$ & $28.0[19.0,39.5]$ & 0.626 \\
\hline
\end{tabular}

$\mathrm{IQR}$, interquartile range; $\mathrm{CV}$, cardiovascular; $\mathrm{CHF} / \mathrm{AMI} / \mathrm{Arrhy}$, congestive heart failure/acute myocardial infarction/arrhythmia; Abd surgery, abdominal surgery; ENT, ear nose throat; APACHE II, Acute Physiology and Chronic Health Evaluation II; MV, mechanical ventilation; ICU, intensive care unit; LOS, length of stay

\section{3-2. Multivariable Analysis}

Figure 3 shows the Forrest plot of odds ratio and 95\% confidence interval for each covariate after adjustment with multivariable analysis. High HADS-D score was an independent factor for a high probability of poor appetite (OR, 1.20; 95\%CI, 1.14-1.28; $\mathrm{p}=0.00)$. Age was not significantly associated with a poor appetite. Additionally, Figure 4 shows 
the relationship between the severity of depressive symptoms and probability of appetite loss after adjusting for covariates.

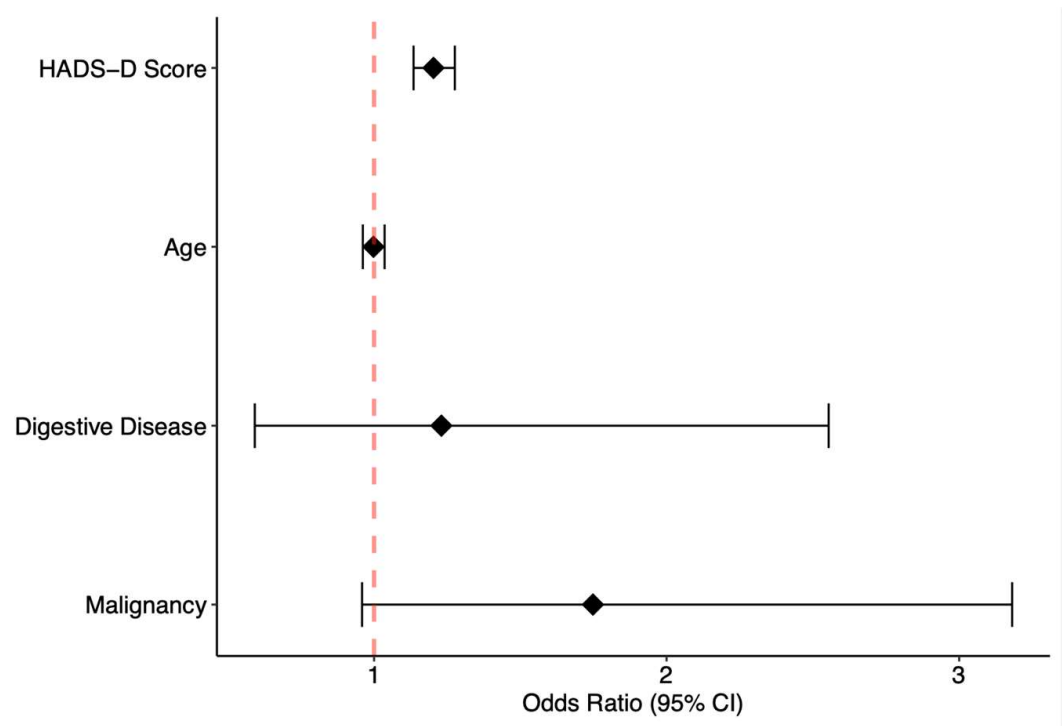

Figure 3. The results of multilevel generalized linier model with binomial family and logit link. Forrest plot of odds ratio and $95 \%$ for adjusted variables.

HADS-D shows the odds ratio for each one-point change in the score. Similarly, the odds ratio for a one-year change in age is shown. HADS-D, Hospital Anxiety and Depression Scale-Depression

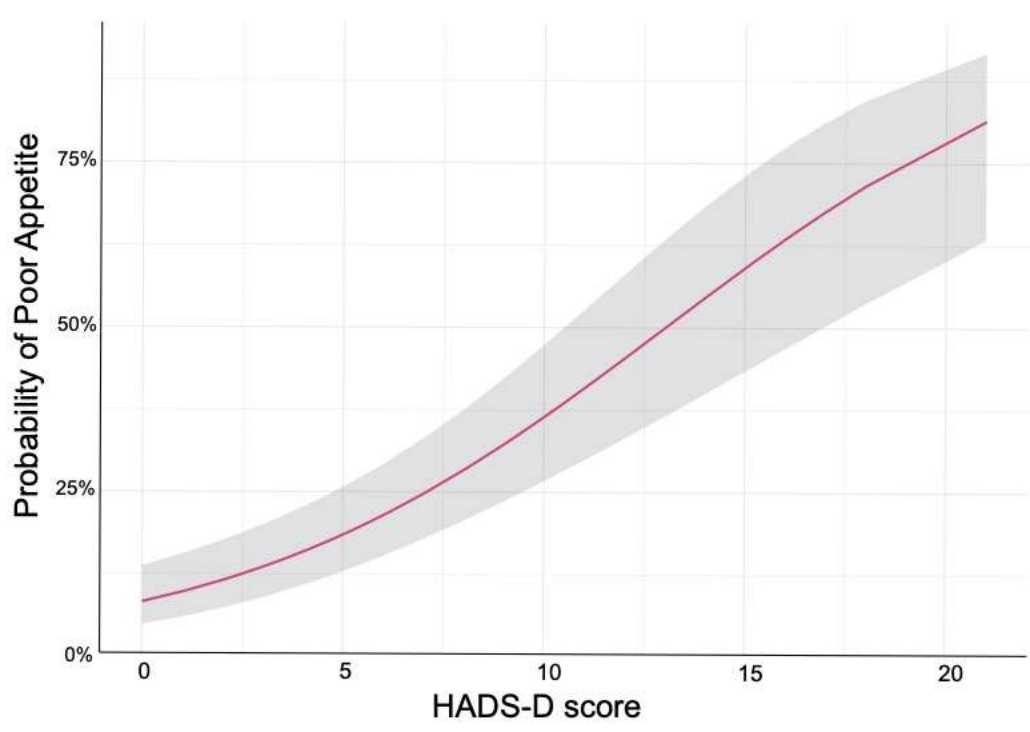


Figure 4. The relationship between severity of depression and risk of poor appetite after adjusting for the covariates age, HADS-D score, digestive disease, and malignancy, using a multilevel generalized linier model with binomial family and logit link.

Grey areas show 95\% confidence intervals. HADS-D is a subscale of the HADS that assesses depressive symptoms. A high HADS-D score indicates a high number of symptoms. Poor appetite was defined as a SNAQ score <14. HADS, Hospital Anxiety and Depression Scale; HADS-D, Hospital Anxiety and Depression Scale - Depression subscale.

\section{Discussion}

\subsection{Summary of main findings}

We conducted a secondary analysis to clarify the prevalence of appetite loss and the relationship between appetite loss and severity of depression 12 months after intensive care in the older population. One of the four patients experienced significant appetite loss, and the severity of depression was an independent factor contributing to appetite loss.

\subsection{Comparison of Current and Previous Results}

In the present study, appetite loss was more common in the post-ICU than in the general community-dwelling older population and was similar to that in rehabilitation units. In one study, in 9496 Japanese older individuals, the prevalence of appetite loss (SNAQ score <14) was 9.8\% [2]. Another report indicated that $24.7 \%$ of the people aged 65 or older in the rehabilitation unit had an SNAQ score $<14$ [11], which was comparable with that reported in our study.

The mechanisms underlying appetite loss after intensive care remain unclear. Poor appetite immediately and 1 week after intensive care was associated with the level of Creactive protein (CRP); however, after 3 months, there was no longer a relationship between anorexia and CRP level [16]. Thus, it is unlikely that the CRP level had an impact on appetite status 1 year after intensive care. The finding that abdominal disease did not affect appetite loss at 1 year in this study is consistent with that reported in previous studies, where subtotal gastric surgery did not cause appetite loss at 1 year [17]. A high SF-36 physical domain was associated with a low risk of appetite loss [18], suggesting that physical disability and low ADL after ICU discharge may have led to appetite loss in this study.

\subsection{Relationship between Poor Appetite and Depression}

High severity of depression may be associated with a high probability of appetite loss in our population. This finding is consistent with that of a previous cross-sectional study, where appetite loss was associated with a high probability of depression in community-dwelling older people and people living in assisted facilities [6,19]. Depression is a common psychological problem and an important co-symptom of decreased appetite and motivation to eat in older adults; however, the underlying mechanism is not well known [20]. A high prevalence of depression has been reported in post-intensive care patients [7], so we should focus on anorexia as well as depression. This may contribute to the improvement in the QoL of patients discharged from the ICU.

\subsection{Strengths and Limitations}

To the best of our knowledge, this is the first study to use a validated tool to investigate appetite status 1 year after ICU discharge; however, some limitations exist. In this study conducted in Japan, the characteristics of ICU patients (i.e., a high proportion of scheduled surgery) reflected the findings. Thus, different populations of patients admitted to the ICU may have led to different results. However, appetite loss appeared to be independent of the severity of illness or reason for ICU admission; thus, we do not consider this aspect to have a significant impact on external validity. In this study, we measured appetite loss using the SNAQ; however, the prevalence rates of actual weight loss, sarcopenia, and frailty were not determined. Further studies are needed to clarify the frequency of sarcopenia and frailty after intensive care in a large prospective cohort. Moreover, we clarified that appetite loss was associated with a high severity of depression; 
however, the causal relationship was unknown because of our study design. A study design that measures one of the factors earlier is needed.

\subsection{Clinical Implications}

We must focus on appetite loss as well as changes in body weight and depressive symptoms in the post-ICU older population. Adequate nutrition counseling in addition to physical examination may be useful for early detection and improvement of nutritional status and could lead to the prevention of sarcopenia.

\section{Conclusion}

Poor appetite is a common finding 12 months after ICU admission. This may lead to sarcopenia and a high mortality rate. Additionally, poor appetite is associated with a high severity of depression. It is necessary to monitor and treat these symptoms when observed after intensive care.

\section{Funding}

This research was funded by JSPS KAKENHI (grant number: 19K10929).Acknowledgments

We thank Mr. Tomoo Sato, Mr. Takeharu Miyamoto, Mr. Yuki Wakabayashi, Ms. Haruka Nakagawa, and Mr. Yuta Ikeda for data collection; Ms. Keiko Wataya for advice concerning mental support for the study participants; and Mr. Fumihide Shinohara for data cleaning support.

The following investigators were involved in the study protocol: Ryuta Indo, Hiroomi Tatsumi, Atsuko Handa, Kazuyo Koori, Ayano Kudo, Kayo Kitaura, Etsuko Moro, Shin Nunomiya, Ouchi Akira, Masako Sato, Yoshiaki Inoue, Etsuko Tsukioka, Yasuhiro Kishi, Chiaki Sakamoto, Kohei Matsuba, Hiroki Isonishi, Ikumi Kobashi, Miki Toyoshima, Masahiro Yamane, Yumi Kajiyama, and Yoshihumi Heshiki.

\section{Author Contributions}

Conceptualization, T. U., H.S., and Y.M. Formal Analysis, T.U.; Funding acquisition, T.U.; Methodolody, T.U. and H.S.; Project administration, T.U.; Validation, T.U., Investigation, S.U., T.T., T.Y., Y.S, M.H., T.K., Y.F., T.N., M.K., M.S., J.H., Y.S, and Y.M.; Visualization, T.U.; Formal Analysis, T. U.; Writing-original draft, T.U. and A.S.; Writing -review \& editing, T.U., A.S., S.U., T.T., T.Y., Y.S, M.H., T.K., Y.F., T.N., M.K., M.S., J.H., Y.S, and Y.M.

\section{Institutional Review Board Statement}

The study was conducted in accordance with the guidelines of the Declaration of Helsinki and approved by the Institutional Review Board of Sapporo City University (No. 1927-1 and August 30 ${ }^{\text {th }}$, 2019). Additionally, ethical approval was obtained from the ethical committees of all participating institutions.

\section{Data Availability Statement}

The data that support the findings of this study are available upon request from the corresponding author (T.U.). The data are not publicly available because they contain information that can compromise the privacy of the research participants.

\section{Conflicts of Interest}

The authors declare no conflict of interest.

\section{REFERENCES}

1. Chapman, I.M. The anorexia of aging. Clin. Geriatr. Med. 2007, 23, 735-56, v. 
2. Tsutsumimoto, K.; Doi, T.; Nakakubo, S.; Kim, M.; Kurita, S.; Ishii, H.; Shimada, H. Association between anorexia of ageing and sarcopenia among Japanese older adults. J. Cachexia Sarcopenia Muscle 2020, 11, 1250-1257.

3. Giezenaar, C.; Chapman, I.; Luscombe-Marsh, N.; Feinle-Bisset, C.; Horowitz, M.; Soenen, S. Ageing Is Associated with Decreases in Appetite and Energy Intake--A Meta-Analysis in Healthy Adults. Nutrients 2016, 8, doi:10.3390/nu8010028.

4. $\quad \mathrm{Xu}$, J.; Wan, C.S.; Ktoris, K.; Reijnierse, E.M.; Maier, A.B. Sarcopenia Is Associated with Mortality in Adults: A Systematic Review and Meta-Analysis. Gerontology 2021, 1-16.

5. Malmgren, J.; Waldenström, A.-C.; Rylander, C.; Johannesson, E.; Lundin, S. Long-term health-related quality of life and burden of disease after intensive care: development of a patient-reported outcome measure. Crit. Care 2021, $25,82$.

6. Lee, J.S.; Kritchevsky, S.B.; Tylavsky, F.; Harris, T.B.; Ayonayon, H.N.; Newman, A.B. Factors associated with impaired appetite in well-functioning community-dwelling older adults. J. Nutr. Elder. 2006, 26, 27-43.

7. Rabiee, A.; Nikayin, S.; Hashem, M.D.; Huang, M.; Dinglas, V.D.; Bienvenu, O.J.; Turnbull, A.E.; Needham, D.M. Depressive Symptoms After Critical Illness: A Systematic Review and Meta-Analysis. Crit. Care Med. 2016, 44, $1744-1753$.

8. Unoki, T.; Sakuramoto, H.; Uemura, S.; Tsujimoto, T.; Yamaguchi, T.; Shiba, Y.; Hino, M.; Kuribara, T.; Fukuda, Y.; Nagao, T.; et al. Prevalence of and risk factors for post-intensive care syndrome: Multicenter study of patients living at home after treatment in 12 Japanese intensive care units, SMAP-HoPe study. PLoS One 2021, 16, e0252167.

9. Wilson, M.-M.G.; Thomas, D.R.; Rubenstein, L.Z.; Chibnall, J.T.; Anderson, S.; Baxi, A.; Diebold, M.R.; Morley, J.E. Appetite assessment: simple appetite questionnaire predicts weight loss in community-dwelling adults and nursing home residents. Am. J. Clin. Nutr. 2005, 82, 1074-1081.

10. Nakatsu, N.; Sawa, R.; Misu, S.; Ueda, Y.; Ono, R. Reliability and validity of the Japanese version of the simplified nutritional appetite questionnaire in community-dwelling older adults. Geriatr. Gerontol. Int. 2015, 15, $1264-1269$.

11. Shimizu, A.; Fujishima, I.; Maeda, K.; Murotani, K.; Inoue, T.; Ohno, T.; Nomoto, A.; Ueshima, J.; Ishida, Y.; Nagano, A.; et al. Accuracy of the Simplified Nutritional Appetite Questionnaire for Malnutrition and Sarcopenia Screening among Older Patients Requiring Rehabilitation. Nutrients 2021, 13, doi:10.3390/nu13082738.

12. Zigmond, A.S.; Snaith, R.P. The hospital anxiety and depression scale. Acta Psychiatr. Scand. 1983, 67, 361-370.

13. Jutte, J.E.; Needham, D.M.; Pfoh, E.R.; Bienvenu, O.J. Psychometric evaluation of the Hospital Anxiety and Depression Scale 3 months after acute lung injury. J. Crit. Care 2015, 30, 793-798.

14. Hatta, H.; Higashi, A.; Yashiro, H.; Kotaro, O.; Hayashi, K.; Kiyota, K.; Inokuchi, H.; Ikeda, J.; Fujita, K. A Validation of the Hospital Anxiety and Depression Scale. Jpn J Psychosom Med 1998, 38, 309-315.

15. Bell, M.L.; Fairclough, D.L.; Fiero, M.H.; Butow, P.N. Handling missing items in the Hospital Anxiety and Depression Scale (HADS): a simulation study. BMC Res. Notes 2016, 9, 479.

16. Merriweather, J.L.; Griffith, D.M.; Walsh, T.S. Appetite during the recovery phase of critical illness: a cohort study. Eur. J. Clin. Nutr. 2018, 72, 986-992.

17. Jeon, T.Y.; Lee, S.; Kim, H.H.; Kim, Y.J.; Lee, J.G.; Jeong, D.W.; Kim, Y.J. Long-term changes in gut hormones, appetite and food intake 1 year after subtotal gastrectomy with normal body weight. Eur. J. Clin. Nutr. 2010, 64, 826-831.

18. Acar Tek, N.; Karaçil-Ermumcu, M.Ş. Determinants of Health Related Quality of Life in Home Dwelling Elderly Population: Appetite and Nutritional Status. J. Nutr. Health Aging 2018, 22, 996-1002.

1. 19. 19. Engel, J.H.; Siewerdt, F.; Jackson, R.; Akobundu, U.; Wait, C.; Sahyoun, N. Hardiness, depression, and emotional well-being and their association with appetite in older adults. J. Am. Geriatr. Soc. 2011, 59, 482-487.

2. 20. Raison, C.L.; Capuron, L.; Miller, A.H. Cytokines sing the blues: inflammation and the pathogenesis of depression. Trends Immunol. 2006, 27, 24-31. 